\title{
Biopsychosocial predictors of suicide risk in patients with interstitial cystitis/bladder pain syndrome
}

\author{
Dean A. Tripp, PhD'; Joel Dueck, BSc'; Ronald R. Holden, PhD'; Julia Moreau, BSc'; R. Christopher Doiron, MD'; \\ J. Curtis Nickel, $M D^{3}$
}

'Departments of Psychology, Anesthesia, Urology, Queen's University, Kingston, ON, Canada; ${ }^{2}$ Department of Psychology, Queen's University, Kingston, ON, Canada; ${ }^{3}$ Department of Urology, Queen's University, Kingston, ON, Canada

Cite as: Tripp DA, Dueck J, Holden RR, et al. Biopsychosocial predictors of suicide risk in patients with interstitial cystitis/bladder pain syndrome. Can Urol Assoc J 2021;15(12):E630-6. http://dx.doi.org/10.5489/cuaj.7337

Published online June 22, 2021

\section{Abstract}

Introduction: The impact of interstitial cystitis/bladder pain syndrome (IC/BPS) is prevalent and severe. Studies examining the IC/BPS prevalence and predictors of suicide risk are limited by their lack of theoretically relevant suicide research variables. This research reports suicide risk prevalence and its biopsychosocial predictors for a community IC/BPS sample.

Methods: Self-identified female patients suffering from IC/BPS $(\mathrm{n}=813 ; 18-80$ years, mean 46.60, standard deviation [SD] 14.10) recruited from online IC/BPS support groups completed measures of demographic, pain, symptoms, and psychosocial variables. Descriptive statistics, correlations, and multivariable logistic regressions examined prevalence, variable associations, and suicide risk prediction.

Results: Suicide risk prevalence was $38.1 \%$. Suicide risk was associated with greater odds for exposure to suicide, psychache, hopelessness, and perceived burdensomeness to others. Further, examining suicide risk by levels of pain showed that exposure to suicide and hopelessness were consistent suicide risk predictors across pain levels; psychache for lower levels of pain, depression in moderate levels of pain, and perceived burdensomeness in moderate and severe pain levels.

Conclusions: The high prevalence of suicide risk is alarming and signifies an imperative for recognizing this risk within the IC/BPS population. The identified psychosocial risk factors may be used in refining screening and treatment, and in directing future IC/ BPS research.

\section{Introduction}

Suicide prediction and prevention have not significantly improved over the past several decades. ${ }^{1}$ Suicide is one of the most extreme outcomes associated with interstitial cyst- itis/bladder pain syndrome (IC/BPS). The prevalence of suicidal thinking in IC/BPS samples was reported at $11 \%$, with marital status and depression predicting worse outcomes. ${ }^{2}$ More current IC/BPS tertiary care data reported a $23 \%$ endorsement of suicidal thinking, predicted by helplessness and depression over and above pain, and IC/BPS symptoms. ${ }^{3}$ Evolving IC/BPS suicide risk research must expand to include relevant suicide risk predictors, such as unemployment, ${ }^{2,4,5}$ marital status, ${ }^{2}$ comorbid insomnia, ${ }^{6}$ psychosocial variables of family suicide history/exposure, ${ }^{5}$ and the specific construct "psychache," defined as the chronic mental pain that leads individuals to seek permanent escape. ${ }^{7}$ Further, hopelessness, thwarted belongingness (i.e., thoughts of loneliness and a lack of reciprocal care), and perceived burdensomeness (i.e., perceiving oneself a liability to others) are also critical theoretically supported variables in predicting suicide risk. ${ }^{4}$

Our study objectives were to determine a point-prevalence estimate for suicide risk in female patients with IC/ BPS, and to statistically predict such risk by pain level using various clinically relevant biopsychosocial variables.

\section{Methods}

\section{Participants}

Participants self-identifying with a diagnosis of IC/BPS were recruited from online IC/BPS support groups and completed online questionnaires. All qualifying participants were female, over the age of 18 years, and were able to read and write in English. Exclusion criteria included: ongoing symptomatic urethral strictures, ongoing neurological disease or disorder affecting the bladder or bowel fistula, history of cystitis caused by tuberculosis, radiation therapy or cytoxan/ cyclophosphamide therapy, having undergone augmentation cystoplasty or cystectomy, an active autoimmune or infectious disorder (e.g., Crohn's disease or ulcerative colitis, lupus, rheumatoid arthritis, multiple sclerosis, or HIV), history of cancer (with the exception of skin cancer), pres- 
ence of a major psychiatric disorder or other psychiatric or medical issues that would interfere with study participation (e.g., dementia, psychosis, upcoming major surgery), or severe cardiac, pulmonary, renal, or hepatic disease. These are the same exclusion criteria used by Sutcliffe et al to ensure that pain reported by IC patients is not attributable to other causes. ${ }^{8}$ Additionally, to assess participants as currently suffering from IC/BPS, the RAND Interstitial Cystitis Epidemiology (RICE) Case Definition Questionnaire was used to exclude 192 participants who had not experienced bladder pain in at least three of the previous six months, leaving a final sample of 813 participants with current consistent bladder pain.

\section{Demographic and clinical variables}

Participant age, gender, ethnicity, country of origin, education, relationship status, occupational status, diagnosis, suicide exposure (i.e., "Do you know a family member or other person that has died by suicide?"), presence of insomnia, and smoking habits were collected.

\section{Diagnosis}

The RICE Case Definition Questionnaire assessed the validity of participants' self-reported IC/BPS diagnosis. ${ }^{9}$ The RICE Questionnaire consists of five items used to classify IC/BPS patients according to pain and urgency criteria. Item 1 contains three questions that ask the patient if they have: A) ever experienced pelvic/bladder pain; B) experienced this pain for the majority of any three months out of the previous six months; and C) experienced this pain for the majority of the previous three months. Items 2,4 , and 5 pertain to urination urgency in the previous three months, with item 2 asking if the patient experienced this feeling at any point in the previous three months, item 4 asking if this urgency gets better/worse/stays the same after urination, and item 5 asking the average daily frequency of urination. Item 3 pertains to perceived cause of urination urgency, whether it is due to pain/discomfort in the pelvic area or due to fear of wetting oneself. Items are scored such that endorsement of parts B and C of item 1 as "yes" categorize patients as "currently experiencing pelvic/bladder pain;" endorsement of item 2 as "yes," in addition to item 3 as "pain, pressure or discomfort" and/or item 5 as $\geq 10$, categorize patients as "experiencing urgency."

\section{Urological symptoms}

The O'Leary-Sant Interstitial Cystitis Symptom and Problem Indices (ICSI; ICPI) is a self-report questionnaire of four items assessing the frequency of specific IC symptoms and four items assessing frequency of urination, urination with little physical warning, nocturia, and bladder pain, and how problematic those symptoms were. ${ }^{10}$

\section{Pain}

Two domains assessing an individual's pain over the past two weeks used numeric pain rating (NPR) scales. Domain 1 assessed average, highest, and lowest pain from 0 (no pain) to 10 (worst pain I've ever had). Domain 2 asked whether they experienced pain flares, the frequency, average duration, the severity of and distress caused by these flares from 0 (none) to 10 (the worst possible severity/distress).

\section{Depressive symptoms}

The Patient Health Questionnaire-9 is a reliable, self-report measure that consists of nine items assessing depression within the past two weeks using ratings from 0 (not at all) to 3 (nearly every day). ${ }^{11} \mathrm{~A}$ total was calculated by summing items and used as a depression index; higher scores indicate greater depression.

\section{Pain catastrophizing}

The Pain Catastrophizing Scale is a reliable, self-report measure assessing appraisals of pain experiences using 13 items ranging from 0 (not at all) to 4 (all the time). ${ }^{12}$ The items sum, providing a total and subscales (rumination, magnification, helplessness); higher scores indicate greater catastrophizing.

\section{Hopelessness}

The State Hopelessness Scale (SHS) is a reliable, self-report measure of 10 items assessing pessimism about the future. ${ }^{13}$ Participants were asked how much they agreed with statements from 1 (strongly disagree) to 4 (strongly agree) in the past week. Summed items create a total; higher scores indicate greater hopelessness.

\section{Psychache}

The Psychache Scale (PAS) is a reliable self-report measure of 13 items assessing aspects of psychache (free-floating, nonsituation-specific anguish, hurt, angst, humiliation, or internal perturbation that leads individuals to seek permanent escape). ${ }^{14}$ Items were rated from 0 (never) to 5 (always) and summed for total scores; higher scores indicate greater psychache.

\section{Social support}

Social support was assessed using a single numeric rating scale item asking, "How much do you feel supported by others in your life (family, friends, significant other, cowork- 
ers, doctors, etc.)?" Support was described as any type of support (emotional, practical, companion, etc.). Participants were asked to rate their level of support on a rating scale from 0 (no support at all) to 10 (complete support).

\section{Interpersonal needs}

The Interpersonal Needs Questionnaire-10 (INQ-10) is a reliable self-report measure of 10 items assessing the role a participant plays in their social circle in two areas: thwarted belongingness and perceived burdensomeness. ${ }^{15,16}$ Participants respond to items using a rating scale from 1 (not at all true for me) to 7 (always true for me). Thwarted belongingness assesses loneliness and lack of reciprocal care from one's social circle. Perceived burdensomeness evaluates selfhate and perceiving oneself as a liability to others; higher scores indicate greater loneliness or perceived burden.

\section{Suicide risk}

The Suicidal Behaviors Questionnaire-Revised (SBQ-R) is a four-item questionnaire. ${ }^{17}$ Item 1 assesses lifetime suicidality (suicidal thoughts and actions), and participants were asked to rate their experiences on a rating scale from 1 (never) to 6 (I have attempted to kill myself, and really hoped to die). Item 2 assesses suicidal ideation in the past year, and participants were asked to rate the frequency of ideation on a rating scale from 1 (never) to 5 (very often). Item 3 assesses lifetime suicide plans and participants were asked to rate frequency and intent using a rating scale from 1 (no) to 5 (yes, more than once, and really wanted to do it). Item 4 assesses self-reported future risk of suicide, and participants were asked to rate the likelihood that they would commit suicide in the future using a rating scale from 0 (never) to 6 (very likely). As usually assessed, a total suicide risk score was calculated summing the weighted items. Scores $>6$ and $>7$, respectively, indicate clinically significant suicide risk compared to general and psychiatric inpatient populations.

\section{Procedure}

This study recruited participants from January 2016 until January 2017 after clearance was acquired from the university research ethics board (REB). Recruiting occurred through online patient support groups (i.e., the Interstitial Cystitis Network and Interstitial Cystitis Association). Participants accessed the questionnaires from the support group's webpages where they consented to participate on a secure, online platform. Questionnaire completion was approximately 30-40 minutes. All participants were asked to complete the questionnaires and, as per REB ethical requirements, all participants were permitted to refuse answering select questions as they desired.

\section{Data analysis}

Missing values and outliers' analyses used IBM SPSS, ${ }^{18}$ with missing values described as random. No participant omitted $>20 \%$ of any measure, thus none were excluded. Participants missing values were replaced with the mean sample score of the item. ${ }^{19}$ Suicide risk prevalence (SBQ-R) was assessed by splitting the sample using the general population and inpatient population cutoffs. All variables that correlated significantly $(p<0.05)$ with suicide risk were employed in multivariable logistic regressions. The sample was divided by pain level into three groups for analyses. Pain groups were created by using low, moderate, and high pain based on the average NPR scores, mirroring previous research in pain ratings of mild (0-4.5), moderate (4.6-7.4), or severe pain (7.5-10) splits. This was an important step, allowing for the multivariable logistic regression analyses of suicide risk by pain levels.

\section{Results}

\section{Participants}

A total of 1005 individuals participated. Participants $(n=813)$ ranged in age from 1-80 years (mean [M] 46.60, standard deviation [SD] 14.10). Participants were predominantly Caucasian (93.1\%), from North America (90.1\%), and living with a spouse/partner (76.1\%). Furthermore, $45.9 \%$ were currently employed and $51.9 \%$ possessed a post-secondary degree. Mean symptom scores were calculated for the ICSI (M 13.12, SD 3.91) and the ICPI (M 11.41, SD 3.22).

\section{Prevalence of suicide risk}

Using the adult general population SBQ-R cutoff created an at-risk group ( $\mathrm{n}=310, \mathrm{M} 9.73, \mathrm{SD} 2.65)$ and a not at-risk group ( $n=503, M 3.96$, SD 1.11), with $38.1 \%$ of the sample meeting the suicide risk threshold. Using a more conservative cutoff score of an adult inpatient psychiatric population, $28.7 \%(n=233)$ reported risk for suicide (Fig. 1). Further, we noted the elevated endorsement of $31.1 \%$ for a single suicidal thought item as employed in older studies (e.g., $11 \%)^{2}$

\section{Evaluation of statistical predictors}

Correlations of all variables with suicide risk were in the appropriate direction. Strong correlations were found between average, highest, and lowest pain $(r \geq 0.74, p \leq 0.01)$, ICPI and ICSI total scores $(r=0.81, p<0.01)$, pain and flare distress $(r=0.88, p<0.01)$, and depression and psychache scores $(r=0.71, p<0.01)$. Due to multicollinearity concerns, ICPI and ICSI scores were combined as a total O'Leary- 


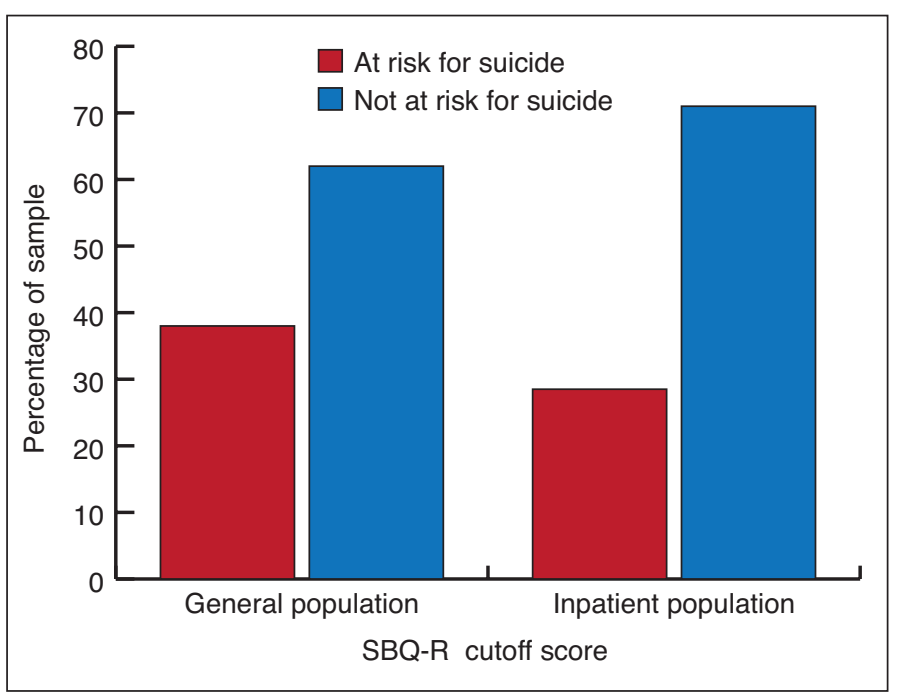

Fig. 1. Percentage of participants in the sample at risk for suicide risk using cut-off scores of $>6$ (adult general population) and $>7$ (adult inpatient population). SBQ-R: Suicidal Behaviors Questionnaire - revised.

Sant Index score. Both depression and psychache scores remained as separate predictors, because they are correlated but distinct factors. ${ }^{20}$

\section{Logistic regression for suicide risk group}

Binary logistic regression analysis was used to determine risk of suicide for the entire sample $(n=813)$ (Table 1$)$. The regression identified $76 \%$ of cases $\left(\chi^{2}(1)=7240.30, p<0.01\right)$. In this model of suicide risk, the predictors of greater risk included a previously reported exposure to suicide (odds ratio OR 2.71, 95\% confidence interval $[\mathrm{Cl}] \mathrm{Cl} 1.84-4.01)$, and the greater presence of psychological factors, such as psychache (i.e., psychological pain) (OR 1.04, 95\% Cl 1.02-1.07), greater hopelessness (OR 1.12, 95\% Cl 1.06-1.17), and more perceptions that the participant was a burden to others (i.e., perceived burdensomeness; OR 1.07, 95\% Cl 1.03-1.11). Interestingly, greater pain catastrophizing was not associated with greater suicide risk in the expected direction (OR $0.978,95 \% \mathrm{Cl} 0.95-0.99)$. An OR of $<1$ means that the first group was less likely to experience the event. This is difficult to quantify because an OR value below 1.00 may not be directly interpretable but suggests that the suicide risk group experienced less pain catastrophizing, in direct contrast to the zero-order correlations that show pain catastrophizing was associated with greater suicide risk $(r=0.13, p<0.05)$.

Logistic regression for suicide risk group in low, moderate, and severe pain As shown in Table 2, low, moderate, and high pain groups were based on the average NPR scores, mirroring previous research in pain ratings of mild (0-4.5), moderate (4.6-7.4), or severe pain (7.5-10). ${ }^{21}$ In this sample, scores from 0-4 represented low $(n=217, M 3.03, S D 1.12), 5-6$ moderate $(=267, M 5.58, S D 0.50)$, and $7-10$ severe pain $(n=329$,
Table 1. Logistic regression predictors for suicide risk for overall sample $(\mathbf{N}=\mathbf{8 1 3})$

\begin{tabular}{lcccc}
\hline Predictor variables & Wald & $\mathbf{p}$ & OR & Cl (95\%) \\
\hline Age & 0.44 & 0.506 & 1.00 & $0.98-1.01$ \\
Marital status & 2.61 & 0.106 & 1.40 & $0.93-2.09$ \\
Exposure to suicide & $\mathbf{2 5 . 1 6}$ & $\mathbf{0 . 0 0 0}$ & $\mathbf{2 . 7 1}$ & $\mathbf{1 . 8 4 - 4 . 0 1}$ \\
Insomnia & 1.92 & 0.166 & 1.30 & $0.90-1.86$ \\
Social support & 0.12 & 0.735 & 0.99 & $0.92-1.07$ \\
Average pain & 1.31 & 0.253 & 0.94 & $0.84-1.05$ \\
Flare severity & 0.05 & 0.827 & 1.01 & $0.95-1.07$ \\
O'Leary-Sant Index & 1.12 & 0.290 & 0.98 & $0.95-1.02$ \\
Catastrophizing & $\mathbf{1 0 . 2 5}$ & $\mathbf{0 . 0 0 1}$ & $\mathbf{0 . 9 7}$ & $\mathbf{0 . 9 5 - 0 . 9 9}$ \\
Psychache & $\mathbf{1 1 . 4 2}$ & $\mathbf{0 0 . 0 0 1}$ & $\mathbf{1 . 0 4}$ & $\mathbf{1 . 0 2 - 1 . 0 7}$ \\
Depression & 2.23 & 0.136 & 1.03 & $0.99-1.08$ \\
Hopelessness & $\mathbf{2 0 . 8 5}$ & $\mathbf{0 . 0 0 0}$ & $\mathbf{1 . 1 2}$ & $\mathbf{1 . 0 6 - 1 . 1 7}$ \\
Perceived burden & $\mathbf{1 4 . 1 1}$ & $\mathbf{0 . 0 0 0}$ & $\mathbf{1 . 0 7}$ & $\mathbf{1 . 0 3 - 1 . 1 1}$ \\
Thwarted belongingness & 1.34 & 0.246 & 1.02 & $0.99-1.05$ \\
\hline
\end{tabular}

Bolded text indicates significant predictors. Cl: confidence interval; OR: odds ratio.

$M 7.86$, SD 1.01). The overall series of results were in the expected directions. The low pain regression $\left(\chi^{2}(14)=66.117\right.$, $\mathrm{p}<0.00 ; 76.5 \%$ identified) showed greater exposure to suicide (OR 2.27, 95\% Cl 1.06-4.86), greater insomnia (OR $2.39,95 \% \mathrm{Cl} 1.05-5.43)$, psychache (OR $1.07,95 \% \mathrm{Cl}$ 1.01-1.13), and hopelessness (OR 1.17, 95\% Cl 1.05-1.30) acted as predictors for greater suicide risk. For moderate pain $\left(\chi^{2}(14)=89.329, p<0.00 ; 78.7 \%\right.$ identified $)$, an exposure to suicide (OR 2.66, 95\% Cl 1.31-5.39), greater depression (OR 1.08, 95\% Cl 1.01-1.16), hopelessness (OR 1.09, 95\% $\mathrm{Cl} 1.00-1.18$ ), and greater perceived burdensomeness (OR $1.08,95 \% \mathrm{Cl} 1.01-1.16)$ were predictors for greater suicide risk. For high pain $\left(\chi^{2}(14)=131.978, p<0.00 ; 77.2 \%\right.$ identified), not living with a partner (OR $0.46,95 \% \mathrm{Cl} 0.24-0.87$ ), exposure to suicide (OR 3.13, 95\% Cl 1.67-5.88), hopelessness (OR 1.13, 95\% Cl 1.05-1.22), and perceived burdensomeness (OR 1.07, 95\% Cl 1.02-1.12) were predictors. As in the previous analyses, the pain catastrophizing index (OR 0.95, 95\% Cl 0.92-0.99) suggests that the suicide risk group experienced less pain catastrophizing.

\section{Discussion}

A $38.1 \%$ prevalence of suicide risk - a value greater than estimates in chronic back pain $(19 \%)$ and patients with wide-ranging chronic pain issues $(14 \%)$ - is alarming. ${ }^{22,23}$ Further, $31.1 \%$ of this sample also endorsed a one-item thoughts of suicide assessment that has been used in older IC/BPS research, reporting a rate of $11 \%{ }^{2,3}$

Examining the overall sample regression, predictors such as exposure to suicide, psychache, hopelessness, and perceived burdensomeness predicted suicide risk. It was also noted that IC/BPS symptoms (i.e., O'Leary-Sant Index) did not predict suicide risk. Hepner et al found a similar effect, 
Tripp et al

\begin{tabular}{|c|c|c|c|c|c|c|c|c|c|c|c|c|}
\hline \multirow[t]{2}{*}{ Predictor variable } & \multicolumn{4}{|c|}{ Low pain ( $n=217)$} & \multicolumn{4}{|c|}{ Moderate pain $(n=267)$} & \multicolumn{4}{|c|}{ Severe pain $(n=329)$} \\
\hline & Wald & $\mathbf{p}$ & OR & Cl (95\%) & Wald & $\mathbf{p}$ & OR & Cl (95\%) & Wald & $\mathbf{p}$ & OR & Cl (95\%) \\
\hline Age & 2.06 & 0.151 & 0.98 & $0.96-1.01$ & 0.18 & 0.668 & 1.01 & $0.98-1.03$ & 0.00 & 0.954 & 1.00 & $0.98-1.02$ \\
\hline Marital status & 0.91 & 0.340 & 1.53 & $0.64-3.69$ & 0.57 & 0.449 & 0.761 & $0.38-1.54$ & 5.59 & 0.018 & 0.46 & $0.24-0.87$ \\
\hline Exposure to suicide & 4.44 & 0.035 & 2.27 & $1.06-4.86$ & 7.37 & 0.007 & 2.66 & $1.31-5.39$ & 12.73 & 0.000 & 3.13 & $1.67-5.88$ \\
\hline Insomnia & 4.32 & 0.038 & 2.39 & $1.05-5.43$ & 0.34 & .562 & 0.827 & $0.44-1.57$ & 1.51 & .219 & 1.45 & $0.80-02.60$ \\
\hline Social support & 00.68 & 0.409 & 1.07 & $0.91-1.27$ & 0.00 & 00.955 & 1.00 & $0.87-1.15$ & 0.99 & 00.319 & 0.94 & $0.84-1.06$ \\
\hline Average pain & 0.34 & 0.560 & 0.90 & $0.64-1.28$ & 0.02 & 0.893 & 1.04 & $0.56-1.97$ & 0.34 & 0.562 & 0.92 & $0.68-1.23$ \\
\hline Flare severity & 0.57 & 0.452 & 0.96 & $0.85-1.07$ & 0.90 & 0.343 & 1.06 & $0.94-1.18$ & 0.00 & 0.999 & 1.00 & $0.89-1.13$ \\
\hline O'Leary-Sant Index & 0.69 & 0.406 & 0.97 & $0.91-1.04$ & 0.05 & 0.831 & 1.01 & $0.95-1.18$ & 0.82 & 0.365 & .98 & $0.93-1.03$ \\
\hline Catastrophizing & 1.98 & 0.159 & 0.97 & $0.94-1.01$ & 2.95 & 0.086 & 0.97 & $0.93-1.01$ & 7.32 & 0.007 & 00.95 & $0.92-0.99$ \\
\hline Psychache & 5.36 & 0.021 & 1.07 & $1.01-1.13$ & 3.18 & 0.075 & 1.04 & $1.00-1.09$ & 3.30 & 0.070 & 1.03 & $1.00-1.07$ \\
\hline Depression & 0.04 & 0.850 & .99 & $0.91-1.08$ & 4.70 & 0.030 & 1.08 & $1.01-1.16$ & 0.57 & 0.450 & 1.03 & $0.96-1.10$ \\
\hline Hopelessness & 7.91 & 0.005 & 1.17 & $1.05-1.30$ & 4.06 & 0.044 & 1.09 & $1.00-1.18$ & 10.98 & 0.001 & 1.13 & $1.05-1.22$ \\
\hline Perceived burden & 1.59 & 0.207 & 1.07 & $0.96-1.19$ & 4.87 & 0.027 & 1.08 & $1.01-1.16$ & 7.02 & 0.008 & 1.07 & $1.02-1.12$ \\
\hline Thwarted belongingness & 0.54 & 0.465 & 1.03 & $0.96-1.10$ & 0.08 & 0.784 & 1.01 & $0.95-1.07$ & 1.31 & 0.253 & 1.03 & $0.98-1.09$ \\
\hline
\end{tabular}

Bolded text indicates significant predictors. $\mathrm{Cl}$ : confidence interval; OR: odds ratio.

showing that symptom severity does not independently predict likelihood of suicide risk. ${ }^{2}$ These are also the first results to indicate that psychosocial factors, such as hopelessness, perceived burden, and psychache act as independent predictors of IC/BPS suicide risk. In our pain group regressions, pain moderated the profile of psychosocial predictors except for thwarted belongingness, which failed to predict risk, thereby confirming previous pain research. ${ }^{24}$ Perhaps, in this sample, hopelessness is a prime clinical concern, as it predicted risk across all pain groups.

Research on suicide attempters of several ages and clinical severity shows psychache and hopelessness are recurrently and robustly confirmed variables in suicide attempts within suicide theory. ${ }^{25,26}$ Suicide theory also highlights the importance of psychache, in conjunction with hopelessness, for the development of the desire to die, ${ }^{27}$ but no previous research has examined these relations in patients with IC/ BPS or by pain levels. Indeed, psychache predicted suicide risk in low pain and perceived burdensomeness in moderate and severe pain. In our IC/BPS sample, it may be that physical pain moderates the predictors of suicide risk. It may be that the shift to more severe pain may transition the psychache and hopelessness variables into depression, hopelessness, and a perception of being a burden to others, as exemplified in the moderate pain regression. In severe pain, perceived burdensomeness and hopelessness remain associated to higher suicide risk. This association may be supported by other variables, such as a person's self-worth, but longitudinal research is needed to study such effects.

Among the other findings, marital status, exposure to suicide, and insomnia were predictors of suicide risk. Marital status displayed significant value but only for the severe pain group. ${ }^{2}$ As well, for high pain, pain catastrophizing was unexpectedly found to be lower in the group higher in suicide risk. Indeed, pain catastrophizing was significantly correlated with pain and suicide risk in this study. Multicollinearity was ruled out as a cause, and the most likely explanation is that other psychological variables are suppressing the effect of pain catastrophizing in the analyses.

Exposure to suicide was a significant predictor of suicide risk across all pain levels, confirming the strength of this life experience. Indeed, we have concerns about how to support individuals who have been exposed to suicide. Studies show that exposure to suicide can lead to a variety of negative experiences, such as depression, as well as increased risk of suicide ideation; even attempts have been reported. ${ }^{22,28}$ Recent work has highlighted a lack of investigation into mechanisms of the association between exposure to suicide and vulnerability to suicide. As Miklin et al suggest, "suicide exposure" may involve related but also independent events. ${ }^{29}$ For example, the witnessing of another's grief after a suicide is an event, but the impact of such exposure(s) also depends on the way individuals ascribe meaning to the death in the context of their own lives. The current study did not collect variables to help address such issues but future research in IC/BPS and suicide could examine such impacts as predictors in suicide ideation.

The fact that "extent of disease" - in this study, the ICSI/ ICPI score - was not a predictor of suicide risk is an important finding, particularly for clinicians who might use these tools commonly in practice. From a clinical perspective, a community urologist's practice can incorporate these findings into IC/BPS care through increased awareness of the psychosocial suicide risk factors that may trigger suicide assessment(s). Symptoms are of concern for patients but it seems psychosocial factors may be more salient in suicide risk; there is no doubt a recursive relationship between these variables. In regard to the management of suicide risk when 
identified, referral to a multidisciplinary clinic is suggested if available. It is suggested that IC/BPS management should move towards an approach that integrates managing disease activity and psychological well-being concurrently. Clinical interventions in IC/BPS patients with high risk of suicide should target psychosocial predictors of suicidal behavior (i.e., history of suicide exposure, hopelessness, being a selfperceived burden to others, psychache, depressive symptoms) to mitigate their downstream effects.

Limitations of this research include the possibility of selection bias in this online sample. Given this possibility, the alarming rate may suggest that patients engaged in online support activities or groups might be more distressed, but without a comparison group, this will remain speculation. Another study limitation can be the use of self-report methods, which may be considered less reliable than professional assessments; however, self-report remains the gold-standard on patient reporting of distress and depressive symptomotology, making such a limitation unavoidable in this suicide research. Further, online survey responses show strong reliability and validity while acknowledging self-selection biases. ${ }^{30}$ Therefore, we believe that online responses are as valid as in-person, acknowledging they may overestimate effects. This study was also cross-sectional with correlationbased analyses, where causality cannot be established. However, the results are supported by previous IC/BPS suicide research. ${ }^{2,3}$ Suicide risk is an important measure of suicidality, but may not fully predict suicide mortality, and therefore, these figures may provide an overestimation of the likelihood of suicide mortality in this sample. Finally, generalizability of findings should be cautioned due to sample homogeneity.

\section{Conclusions}

The results confirm that suicide risk is a significant concern within the IC/BPS population and work is needed to understand how to address the increased needs of the at-risk women. Suicide risk is more related to psychosocial factors than physical IC/BPS factors. With many suicide risks modifiable by targeted treatment, perhaps a suicide risk identification and prevention strategy in IC/BPS management is justified. In particular, hopelessness, psychache, perceived burdensomeness, and exposure to previous suicide are important predictors to include in developing clinical interventions and future research in IC/BPS.

Competing interests: The authors do not report any competing personal or financial interests related to this work.

This paper has been peer-reviewed.

\section{References}

1. Franklin JC, Ribeiro JD, Fox KR, et al. Risk factors for suicidal thoughts and behaviors: A meta-analysis of 50 years of research. Psychol Bull 2017;143:187-232. https://doi.org/10.1037/bul0000084

2. Hepner KA, Watkins KE, Watkins MN, et al. Suicidal ideation among patients with bladder pain syndrome/ interstitial cystitis. Urology 2012;80:280-5. https://doi.org/10.1016/i.urology.2011.12.053

3. Tripp DA, Nickel JC, Krsmanovic A, et al. Depression and catastrophizing predict suicidal ideation in tertiary care patients with interstitial cystitis/bladder pain syndrome. Can Urol Assoc J 2016;10:383-8. https://doi.org/10.5489/cuai.3892

4. Racine M. Chronic pain and suicide risk: a comprehensive review. Prog Neuropsychopharmacol Biol Psychiatry 2018;87:269-80. https://doi.org/10.1016/i.pnpbp.2017.08.020

5. Van Orden KA, Witte TK, Cukrowicz KC, et al. The interpersonal theory of suicide. Psychol Rev 2010;117:575-600. https://doi.org/10.1037/00018697

6. Calandre EP, Vilchez JS, Molina-Barea R, et al. Suicide attempts and risk of suicide in patients with fibromyalgia: A survey in Spanish patients. Rheumatology (Oxford) 2011;50:1889-93. https://doi.org/10.1093/rheumatology/ker203

7. Shneidman ES. Suicide as psychache: A clinical approach to self-destructive behavior. Northvale, N.J. Rowman \& Littlefield Publishers, 1993.

8. Sutcliffe $S$, Colditz GA, Goodman MS, et al. Urological chronic pelvic pain syndrome symptom flares: Characterization of the full range of flares at two sites in the Multidisciplinary Approach to the Study of Chronic Pelvic Pain (MAPP) Research Network. BJU Int 2014;114:916-25. https://doi.org/10.1111/ bju. 12778

9. Berry SH, Bogart LM, Pham C, et al. Development, validation and testing of an epidemiological case definition of interstitial cystitis/painful bladder syndrome. J Urol 2010;183:1848-52. https://doi.org/10.1016/i.juro.2009.12.103

10. O'Leary MP, Sant GR, Fowler FJ, et al. The interstitial cystitis symptom index and problem index. Urology 1997;49:58-63. https://doi.org/10.1016/S0090-4295(99)80333-1

11. Kroenke K, Spitzer RL, Williams JB. The PHQ-9: Validity of a brief depression severity measure. J Gen Intern Med 2001;16:606-13. https://doi.org/10.1046/i.1525-1497.2001.016009606.x

12. Sullivan MJ, Bishop SR, Pivik J. The pain catastrophizing scale: Development and validation. Psychol Assess 1995;7:524-32. https://doi.org/10.1037/1040-3590.7.4.524

13. Dunn SL, Olamijulo GB, Fuglseth HL, et al. The state-trait hopelessness scale: Development and testing. West J Nurs Res 2014;36:552-70. https://doi.org/10.1177/0193945913507634

14. Holden RR, Mehta K, Cunningham EJ, et al. Development and preliminary validation of a scale of psychache. Can J Behav Sci 2001;33:224-32. https://doi.org/10.1037/h0087144

15. Bryan C, Anestis M. Reexperiencing symptoms and the interpersonal-psychological theory of suicidal behavior among deployed service members evaluated for traumatic brain injury. J Clin Psychol 2011;67:856-65. https://doi.org/10.1002/iclp.20808

16. Joiner TE, Wingate LR, Otamendi A. An interpersonal addendum to the hopelessness theory of depression: hopelessness as a stress and depression generator. J Soc Clin Psychol 2005;24:649-64. https://doi.org/10.1521/iscp.2005.24.5.649

17. Osman A, Bagge CL, Gutierrez PM, et al. The suicidal behaviors questionnaire-revised (SBQ-R): Validation with clinical and nonclinical samples. Assessment 2001;8:443-53. https://doi.org/10.1177/107319110100800409

18. Hayes AF. Introduction to mediation, moderation, and conditional process analysis: A regression-based approach 2nd edition. New York, N.Y. Guilford Press, 2018.

19. Tabachnick BG, Fidell LS. Using multivariate statistics, 5th edition. Boston, Mass. Allyn \& Bacon/Pearson Education, 2007

20. Delisle MM, Holden RR. Differentiating between depression, hopelessness, and psychache in university undergraduates. Meas Eval Couns Dev 2009;42:46-54. https://doi.org/10.1177/0748175609333562

21. Jensen MP, Chen C, Brugger AM. Interpretation of visual analog scale ratings and change scores: A reanalysis of two clinical trials of postoperative pain. J Pain 2003;4:407-14. https://doi.org/10.1016/ S1526-5900(03)00716-8

22. Smith LD, Peck PL, McGovern RJ. Factors contributing to the utilization of mental health services in a rural setting. Psychol Rep 2004;95:435-42. https://doi.org/10.2466/PR0.95.6.435-442

23. Calati R, Bakhiy $\mathrm{CL}$, Artero $\mathrm{S}$, et al. The impact of physical pain on suicidal thoughts and behaviors: Meta-analyses. J Psychiatr Res 2015;71:16-32. https://doi.org/10.1016/i.jpsychires.2015.09.004

24. Wilson KG, Kowal J, Henderson PR, et al. Chronic pain and the interpersonal theory of suicide. Rehabil Psychol 2013;58:111-5. https://doi.org/10.1037/a0031390

25. May AM, Klonsky ED. Assessing motivations for suicide attempts: development and psychometric properties of the inventory of motivations for suicide attempts. Suicide Life Threat Behav 2013;43:532-46. https://doi.org/10.1111/stb.12037 
Tripp et al

26. May AM, $\mathrm{O}^{\prime} B r i e n \mathrm{KH}$, Liu RT, et al. Descriptive and psychometric properties of the inventory of motivations for suicide attempts (IMSA) in an inpatient adolescent sample. Arch Suicide Res 2016;20:476-82. https://doi.org/10.1080/13811118.2015.1095688

27. Klonsky ED, May AM. The three-step theory (3ST): A new theory of suicide rooted in the "ideation-toaction" framework. Int J Cogn Ther 2015;8:114-29. https://doi.org/10.1521/iict.2015.8.2.114

28. Andriessen K, Rahman B, Draper B, et al. Prevalence of exposure to suicide: A meta-analysis of populationbased studies. J Psychiatr Res 2017;88:113-20. https://doi.org/10.1016/i.jpsychires.2017.01.017

29. Miklin $S$, Mueller AS, Abrutyn $S$, et al. What does it mean to be exposed to suicide?: suicide exposure, suicide risk, and the importance of meaning-making. Social Sci Med 2019;233:21-7. https://doi.org/10.1016/i.socscimed.2019.05.019
30. Ramo DE, Liu H, Prochaska JJ. Reliability and validity of young adults' anonymous online reports of marijuana use and thoughts about use. Psychol Addict Behav 2012;26:801-11. https://doi.org/10.1037/ a0026201

Correspondence: Dr. Dean A. Tripp, Departments of Psychology, Anesthesia, Urology, Queen's University, Kingston, ON, Canada; Dean.Tripp@Queensu.ca 